\title{
Graphical modelling for simulation and formal analysis of wireless network protocols
}

\author{
A. Fehnker ${ }^{1}$, M. Fruth ${ }^{2}$, and A. K. McIver ${ }^{3}$ \\ 1 National ICT Australia, Sydney, Australia; ${ }^{\star}$ ansgar@nicta.com \\ 2 School of Computer Science, University of Birmingham, UK; *夫 \\ m.fruth@cs.bham.ac.uk \\ 3 Dept. Computer Science, Macquarie University, NSW 2109 Australia, and National \\ ICT Australia; anabel@ics.mq.edu.au
}

\begin{abstract}
The aim of this research is to enhance performance analysis of wireless networks based on simulation with formal performance analysis.

It is well-known that the performance of protocols for wireless networks, and their ability to tolerate faults arising due to the uncertainties underlying wireless communication, relies as much on the topology of the network as on the protocols' internal algorithms. Many general-purpose simulation tools however do not use realistic models of wireless communication, and indeed results of simulation experiments can differ widely between simulators and often bear scant relation to field experiments [7, $6]$.

On the other hand, whilst model checking can supply more robust and exhaustive measures of performance, as for simulation, it is similarly flawed in that the details of the wireless communication are often overly simplified.

In this paper we propose a graphical specification style, which eases the study of the effect of topologies in performance analysis by visualising both the spatial characteristics of the network as well as critical measures of performance that they imply. Unlike other graphical visualisation tools, our proposal integrates both simulation using the novel Castalia simulator [3] as well as probabilistic model checking using PRISM [8], where we capture the effect of the topology by using probabilistic abstractions to model reception rates.
\end{abstract}

Keywords: Graphical modelling, simulation, lossy communication channels, probabilistic model checking, wireless networks.

\section{Introduction}

Wireless networks comprise devices with limited computing power together with wireless communication. Protocols for organising large-scale activities over these

\footnotetext{
* National ICT Australia is funded through the Australian Government's Backing Australia's Ability initiative, in part through the Australian Research Council.

** This work was in part supported by the EPSRC grant EP/D076625/1
} 
networks must be tolerant to the random faults intrinsic to the wireless medium, and their effectiveness is judged by detailed performance evaluation. One of the major factors impacting on the accuracy of an evaluation method is the mathematical model for the "communication channels" and, especially important, is that it must account for the unexpected disturbances induced by noise and interference amongst close neighbours. Conventional analysis methods rely on simulators $[1,2]$ incorporating some measure of random faults, however simulation in this context suffers from a number of well-documented problems $[7,6]-$ most notable is that accurate channel models validated against physical data do not normally feature. This leads to unrealistic results of performance analyses, which can vary widely between different simulators.

An alternative to simulation is formal modelling and analysis, which is normally ideally suited to investigating complex protocols, and gives access to profiles of performance which exhaustively range over worst- and best-case behaviour. Inclusion of realistic models of wireless communication implies appeal to analytical formulae to determine the effect on performance of the spatial relationships between nodes, such as the distance and density of near neighbours. These context-dependent details however are not easily added to textual-style formal modelling languages, and indeed they militate against a clear and modular specification style.

In this paper we overcome these difficulties by proposing a simple graphical style of specification. We exploit the observations that (a) the distance between and the density of nodes in a network is the major factor impacting on the integrity of wireless communication (together with physical parameters such as transmission strength), and (b) the simplest way to express the crucial spatial relationships is graphically, so that the details of the formal model of communication are transparent to the user and are provided separately.

Besides its simplicity, the graphical style has other benefits in that it allows designers to visualise various performance indicators such as best- or worstcase signal strength between pairs of nodes, or the nodes' individual power consumption. Similarly the critical events occurring in a sample experiment may be "stepped through" in a typical debugging style. Finally — unlike other graphical visualisation tools - it acts as a "bridge" between formal analysis and the more conventional simulation, providing the option to investigate performance using probabilistic model checking, or to carry out more traditional system-wide simulation experiments. In both cases realistic models for wireless communication play a fundamental role.

Our specific contributions are

1. CaVi, a graphical user interface specialised for modelling networks comprising simple wireless nodes. The tool gives immediate access to crucial performance indicators such as signal strength between pairs of nodes;

2. A translation from a CaVi model to either a formal transition-style model suitable for model checking in the PRISM model checker [8] or as input to the recently-developed Castalia simulator [3]. Castalia is novel in that it incorporates an accurate wireless channel model. The PRISM models are the first 
such formal models which take network topology into account. At present both Castalia and PRISM capture only flooding and gossiping protocols [4, $5]$.

3. The option to visualise the network-wide performance metrics calculated from Castalia simulation experiments.

In Sec. 2 we summarise the interference model of wireless communication, and in Sec. 3 we describe how the CaVi graphical tool can visualise the effects of the spatial relationships. We also describe a translation from the graphical representation to Castalia and PRISM.

\section{Wireless networks and lossy communication}

In this section we describe the context of wireless applications, and the challenges that arise for their formal modelling.

In abstract terms a wireless network is a collection of nodes, running one protocol or a combination of protocols that are deployed over a two-dimensional area. The behaviour of the network depends not only on the protocol, but also on the placement of the nodes in the network, and in particular on the interference patterns arising from neighbouring communications. Inspired by other graphical tools [1], we propose a graphical-style of specification whose novelty is that it acts as a uniform modelling language to combine simulation and model checking. In either case, the interference effects disturbing communication are accounted for by the spatial representation, and are converted to reception probabilities in the translation to formal models.

In the next section we summarise the characteristics of wireless communication, which will set the scene for the graphical style of network specification.

\subsection{Interference in wireless networks}

Standard formal modelling of networked systems features both the behaviour of the individual "processors" (in this case "wireless nodes") and an explicit description of the "communication medium" connecting them. The assumption is that if two nodes are "connected" then they are able to send and receive messages without loss.

In reality, whether two nodes can communicate effectively depends on a number of context-specific factors, including the physical distance between the nodes, the signal strength of the sending node, and the extent to which other neighbouring nodes' activities, and those of the receiver, interfere with the sent message.

This complex "interference model" has been studied in depth and analytical formulae have been developed and experimentally validated [9]. Here we are

able to appeal to those formulae to define a convenient conceptual abstraction of communication in terms of the "probability" that a sent message is received, with the probability computed by taking the distance, signal strength and interference of other nodes into account. 
For example given two nodes $A$ and $B$ a distance $d$ apart (see Fig. 1), the probability that $A$ receives a message from $B$ is given by

$$
p_{B}^{A}\left(d, \iota_{B}\right) \hat{=}\left(1-e^{-\gamma_{B}\left(d, \iota_{B}\right) /(1.28)}\right)^{8 f},
$$

where $f$ is the size of the message and $\gamma_{B}$ is the signal-to-noise ratio. The latter is a function of the distance $d$, and the ambient noise $\iota_{B}$. The signal-to-noise ratio is a measure of how much the background noise interferes with the wireless signal. The signal is dominant and the reception probability is high if $\gamma_{B}\left(d, \iota_{B}\right)$ is large.

The ambient $\iota_{B}$ includes effects due to the noise contributed by the signals from nearby nodes. For example, in Fig. 1 if nodes $B, C$ and $D$ all try to send to node $A$, then the mutual interference effects will produce a probability distribution over the message which $A$ actually receives.

Because of the nature of the wireless communication, however, $A$ will receive at most one message. The probability $p_{B}^{A}$ at $(1)$ is the probability that a message from $B$ is received, and that either no message is received from either of the other nodes, or nothing is received at all. If all three nodes $B, C$ and $D$ send then the probability that $A$ receives any message at all is given by the sum $p_{B}^{A}\left(d, \iota_{A}\right)+p_{C}^{A}\left(d^{\prime}, \iota_{C}\right)+p_{D}^{A}\left(d^{\prime \prime}, \iota_{D}\right)$. We note that this sum also takes into account the contribution to the ambient noise generated by each sender.

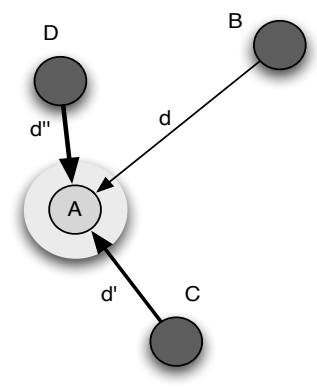

Sender D is closest to receiver A, so its signal is strongest; Sender B's is weakest. All reception probabilities are affected by the others' activities. Here $d, d^{\prime}$ and $d^{\prime \prime}$ are the distances from the senders to the receiver $A$.

Fig. 1. Signal strength varying with distance and interference

\section{CaVi: A graphical specification tool}

$\mathrm{CaVi}$ is a tool which provides specification and analysis support optimised for studying wireless protocols. Its main feature is a graphical interface which eases the task of exploring the effect on performance of different topologies and network parameters. Nodes may be created in a "drag-and-drop" fashion, and the 
properties of individual nodes (such as the available power) may be tuned as necessary. Whilst the network is being created, a user can visualise the optimal "one-hop" signal strength between any pair of nodes, calculated from equation (1). In Fig. 2 we illustrate two examples of how the graphical interface may be used in the design and analysis. The figure shows two panes, with the left being the pane where designers may create and edit a network, and the pane on the right is for visualising the results of simulation experiments.

The pane on the left illustrates visualisation of "one-hop" signal strength by colour-coding the nodes according to probability thresholds calculated from (1). The user may indicate which node is the receiving node (in this case the central node), and the others are assumed to be senders. Colours then differentiate between nodes whose messages will be almost certainly lost (red), or have a good chance of succeeding (green), or merely a variable chance (yellow).

The pane on the right indicates how the events may be viewed as a result of a simulation experiment. The panel on the right gives a list of possible "colourcoded" events (e.g. transmitting, receiving etc.); users may select which events to observe, and the nodes assume the colour of the corresponding event as the simulation is "stepped through".

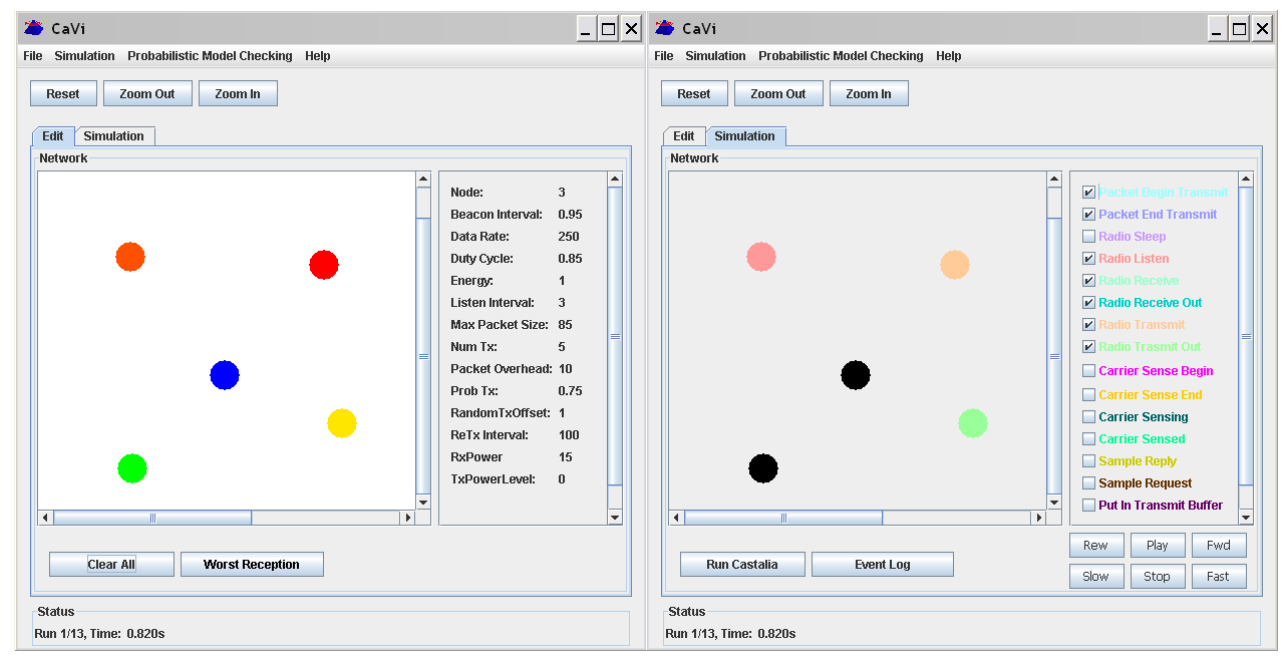

Fig. 2. CaVi:Visualising network performance indicators

Once the network is specified, the graphical representation forms the basis for formal models which take account of the effect of the topology in terms of the reception probabilities. At present we have implemented the automated conversion to a textual format suitable for evaluation directly in the Castalia simulator [3], and as mentioned above the results of experiments may be visualised in various ways described at Fig. 2. We note that the Castalia simulator is a recently-developed simulator whose novelty is a realistic channel/radio model 
which builds on recent work done on modelling of the radio and the wireless channel based on empirically measured data [9].

\subsection{Formal model checking}

One of the major outcomes of this work has been to introduce realistic channel behaviour into formal models for more detailed analysis via probabilistic model checking. We use an abstraction of signal strength in terms of the probability of reception, computed from a formula based on on (1). In Fig. 3 we illustrate the formal template model of a simple node whose only capabilities are that it can receive or send a message, or "do nothing". If in receiving mode $(r e c v=1)$ then the chance that it receives a message is $p_{r}$ which is computed from (1) based on the states of the surrounding nodes. A network is made up of a collection of similar nodes in parallel.

This abstraction of the wireless communication using reception probabilities has been implemented in the PRISM model checker for simple flooding protocols, where the use of the formula rather than expanding the size of the resulting model leads to exceedingly compact models making probabilistic model checking a viable option.

At present we do not have an automated generation of PRISM models from $\mathrm{CaVi}$ - that remains a topic for future research.

$$
\text { Node } \hat{=}\left(\begin{array}{l}
\text { var send, recv: }\{0,1\} \\
\text { tick }:(\text { recv }=1) \rightarrow \text { send, recv }:=1,0 ; \quad p_{r} \oplus \text { skip; } \\
\text { tick }:(\operatorname{send}=1) \rightarrow \text { send, recv: }=0,0 ; \\
\text { tick }:(\operatorname{send}=0 \wedge \text { recv }=0) \rightarrow \text { skip; }
\end{array}\right)
$$

The probability $p_{r}$ is computed as a function of the state dependent on the neighbouring nodes. Here tick is a named event, each "guarded" by a Boolean-function of the state; if any one of the guards is true, then the variables are updated according to the assignments on the right-hand side of the arrow. The probabilistic choice operator $p_{r} \oplus$ means that the left-hand side of the operator is executed with probability $p_{r}$, and the right-hand side with probability $1-p_{r}$.

Fig. 3. A template for a node with parameterised reception probability.

\section{Conclusions and future work}

In this paper we have described a prototype tool which supports a uniform modelling approach optimised for specifying wireless protocols. Its main features include the capabilities to take account of the topology and other parameters of the network which, experiments have shown, have a major impact on the integrity of the communication. The CaVi tool allows the specification of a network via 
a graphical interface, and the automated generation to a format for simulation. Detailed performance indicators may be visualised during specification of the network, as well as the results of subsequent simulation experiments.

The principal difference between $\mathrm{CaVi}$ and other specification tools is the link it provides between simulation and formal model checking. To simplify the details related to the topology in the formal specification task, we use a translation directly to reception probabilities. Those probabilities are calculated according to a validated analytic formula.

An understanding of realistic channel behaviour has suggested some novel approaches to formal verification of wireless protocols, and in the future we hope to incorporate such detailed analyses within the CaVi tool.

For the future we would like to automate the translation from $\mathrm{CaVi}$ to PRISM, making CaVi a truly uniform interface between simulation and model checking. Whilst we do not envisage a translation from a CaVi model of an arbitrary protocol to PRISM, we aim rather to provide a library of templates for certain classes of protocol whose precise behaviour can be defined by a number of parameters, in the same way that models are defined in Castalia.

One of the benefits would be a single "top-level" graphical model for simulation and model checking and the ability to visualise the results obtained from both in a uniform way. Such a "bridging language" would allow "counterexamples" computed via model checking to be validated in the simulator, for example.

In the longer term we would like to expand the repertoire of protocols, and to build up a repository of well-studied templates for Castalia and PRISM patterns.

\section{References}

1. OPNET

http://www .opnet.com/.

2. The network simulator ns- 2 . http://www.isi.edu/nsnam/ns/.

3. A. Boulis. Castalia: A simulator for wireless sensor networks. http://castalia.npc.nicta.com.au.

4. A.Fehnker and P. Gao. Formal verification and simulation for performance analysis of probabilistic broadcast protocols. In $5^{\prime}$ th International Conference, ADHOCNOW, volume 4104 of $L N C S$, pages 128-141. Springer, 2006.

5. A.Fehnker and A. McIver. Formal analysis of wireless protocols. In Proc 2nd International Symposium in Leveraging applications in formal methods, verification and validation, 2006.

6. D. Cavin, Y. Sasson, and A. Schiper. On the accuracy of manet simulators. In Proceedings of the second ACM international workshop on Principles of mobile computing, pages 38-43. ACM Press, 2002.

7. K. Kotz, C. Newport, R.S.Gray, J. Liu, Y. Yuan, and C. Elliott. Experimental evaluation of wireless simulation assumptions. In Proceedings of the th ACM international symposium on Modeling, analysis and simulation of wireless and mobile systems, pages 78-82. ACM Press, 2004.

8. PRISM. Probabilistic symbolic model checker. www.cs.bham.ac.uk/ dxp/prism. 
9. M. Zuniga and B. Krishnamachari. Analyzing the transitional region in low power wireless links. In First IEEE International Conference on Sensor and Ad hoc Communications and Networks (SECON), pages 517-526. IEEE, 2004. 